\title{
Case series of acute nutritional neuropathies after gastric bypass: A Guillain Barré syndrome mimic
}

\author{
Thy P. Nguyen* Akhila Vijayakumar, Suur Biliciler, Kazim Sheikh \\ Department of Neurology, University of Texas Health Science Center at Houston, Houston, USA
}

Received: July 24, 2016

Accepted: October 16, 2016

Online Published: October 28, 2016

DOI: $10.5430 /$ crcp.v4n1p37

URL: http://dx.doi.org/10.5430/crcp.v4n1p37

\begin{abstract}
Gastric bypass procedures for morbid obesity are increasingly common in the United States. Neuropathy following gastric bypass surgeries is estimated at $6 \%$. Most practitioners recognize this as a chronic complication due to nutritional deficiencies. We present three cases of severe, acute, nutritional neuropathy occurring after gastric bypass surgeries.

We retrospectively reviewed charts of three patients with acute to subacute neuropathies following gastric bypass surgeries presenting over one year to UTHealth. Data regarding clinical presentation, electrophysiology, diagnostic studies and outcomes are collected. We identified three patients with acute, disabling, ascending numbness and weakness. All patients had intractable vomiting and significant rapid weight loss. Electrodiagnostic studies revealed axonal sensory-motor neuropathy. Cerebrospinal fluid (CSF) studies did not show albuminocytologic dissociation. Two patients were treated with immunomodulation. Nutritional deficiencies were identified as the etiology in all patients. Further reports and research may prevent unnecessary and costly immunomodulatory treatments.
\end{abstract}

Key Words: Nutritional, Guillain Barré syndrome, Peripheral neuropathy, All neuromuscular diseases, EMG

\section{INTRODUCTION}

Gastric bypass procedures for morbid obesity are increasingly common in the United States. Peripheral neuropathy following these procedures is estimated at $6 \% .^{[1]}$ Most neurologists recognize this as a chronic complication due to nutritional deficiencies. We present three cases of severe, acute, nutritional neuropathic disorder occurring after gastric bypass surgery. We discuss the challenges in distinguishing these cases from Guillain-Barré syndrome (GBS). Since this is a small case series, IRB approval was not required.

\section{Methods}

We retrospectively reviewed charts of three patients with acute neuropathies we encountered in the Department of Neurology at University of Texas Health Sciences Center at Houston following gastric bypass surgeries over one year (2013-2014). As this is a case series of 3 patients treated by different practitioners; treatments, laboratory evaluations, diagnostic testing, follow up examinations and vitamin supplementation were not uniform. None of the patients had surgery at our institution. Each patient had their surgery at a different center. Therefore, neurologic complication rate could not be calculated. Additionally, there was no information regarding protocols for vitamin supplementation for each separate surgical center. Patients were all reportedly compliant with vitamin supplementation, but all developed intractable vomiting. Clinical, laboratory, imaging and electrophysiologic data were collected, when available (see Table $1)$.

*Correspondence: Thy P. Nguyen, MD; Email: thy.p.nguyen@uth.tmc.edu; Address: 6431 Fannin St. MSE R 462 Houston, USA. 
Table 1. Clinical Characteristics

\begin{tabular}{llll}
\hline & Patient 1 & Patient 2 & Patient 3 \\
\hline Progression to Nadir & 6 weeks & 2 weeks & 3 weeks \\
Pain & Yes & Yes & Yes \\
Time Interval & 5 months & 1 year and 9 months & 4 months \\
Rate of Weight loss & $100 \mathrm{lb} / 5$ months & $170 \mathrm{lb} / 8$ months & $100 \mathrm{lb} / 4 \mathrm{months}$ \\
Change in BMI & 15.2 & 35.33 & 14.77 \\
EBW Loss & $84 \%$ & $100 \%$ & $43 \%$ \\
TBW Loss & $38 \%$ & $58 \%$ & $24 \%$ \\
Cranial neuropathy & Absent & Absent & Absent \\
Respiratory compromise & Absent & Absent & Absent \\
Dysautonomia & Absent & Absent & Absent \\
Areflexia & Yes & Legs only & Yes \\
MRC SS NADIR & 36 & 48 & 45 \\
Vitamin B1, Normal 72-222 nmol/L & Low $<2$ & Low 7.3 & Low 20 \\
Vitamin B6 & Low & Normal & Normal \\
Vitamin B12 & Normal & Normal & Normal \\
Vitamin D & Normal & Low & Low \\
Copper & Normal & Low & Normal \\
Treatment & IVIG & IVIG & No days \\
Length of stay & 14 days & 52 & 7 days \\
Follow up MRC SS & 52 & Lost to follow up
\end{tabular}

Note. *Time Interval indicates interval between gastric bypass surgery and onset of symptoms; **Treatment indicates immunomodulatory treatment only, all patients received nutritional replacement; IVIG (intravenous immunoglobulin), MRC SS: Medical research council sum score: This is a sum of muscle grading of bilateral deltoids, biceps, wrist extensors, iliopsoas, quadriceps and tibialis anterior.

\section{Case reports}

\subsection{Case 1}

A 24-year-old woman with Graves disease and morbid obesity underwent gastric bypass (Roux-enY) with complications of intractable vomiting requiring multiple hospitalizations. She also reported severe neuropathic pain during this time. She lost 100 pounds in 5 months, $84 \%$ of her excess body weight (EBW). Five months after the gastric bypass, she presented with rapidly progressive, flaccid, areflexic quadriparesis over a 6 week period. Medical research council sum score (MRC SS) is the sum of the strength muscle grading of bilateral deltoids, elbow flexion, wrist extension, hip flexion, knee extension and dorsiflexion. (A score of 60 would indicate normal strength.) MRC SS was 36 at the patient's nadir and the patient was wheelchair bound. Electrodiagnostic studies showed severe, axonal, sensory-motor polyneuropathy (see Figure 1). Cerebrospinal fluid (CSF) studies did not show albuminocytologic dissociation. Serum thiamine levels were undetectable $(<2 \mathrm{nmol} / \mathrm{L}$, normal range $78-185 \mathrm{nmol} / \mathrm{L}$ ). Thiamine was repleted at $100 \mathrm{mg}$ intravenous (IV) daily for 5 days followed by thiamine $100 \mathrm{mg}$ orally thereafter. She concurrently underwent treatment with intravenous immunoglobulin (IVIG) at a dose of $2 \mathrm{~g} / \mathrm{kg}$ administered over 5 days, for a presumed diagnosis of axonal Guillain Barre syndrome. She did not have significant improvement of strength at discharge. Sub- sequent thiamine serum testing 3 months after discharge was low normal at $81 \mathrm{nmol} / \mathrm{L}$. Thiamine was checked at 6 month intervals while the patient was on oral supplementation. She had slow improvement in her strength over $>12$ months. After 12 months, her MRC SS improved to 52 (see Table 1).

\subsection{Case 2}

A 42-year-old woman with history of complicated gastric bypass requiring multiple, gastrointestinal surgical revisions presented with rapidly progressive ascending paresthesias and weakness over a 2 week period. She had intractable vomiting and diarrhea. She lost 170 pounds over an 8 month period, which was $100 \%$ EBW. Examination showed quadriparesis, absent reflexes in the legs and length-dependent sensory changes. MRC SS was 42 and the patient was wheelchair bound. (Bilateral deltoids, elbow flexion and wrist extension were $4 / 5$ on MRC grading scale. Bilateral hip flexors were $3 / 5$, knee extensors were $4 / 5$ and dorsiflexion was 2/5.) Electrodiagnostic studies showed lengthdependent, axonal, sensory-motor neuropathy. She did not have albuminocytologic dissociation. She was found to have low copper and thiamine. Copper was $34 \mathrm{nmol} / \mathrm{L}$ with a normal range of $72-166 \mathrm{nmol} / \mathrm{L}$. Thiamine was $7.3 \mathrm{nmol} / \mathrm{L}$ with a normal range of $8.1-32.9 \mathrm{nmol} / \mathrm{L}$. Thiamine was repleted with $100 \mathrm{mg}$ IV daily dose followed by indefinite oral 
thiamine $100 \mathrm{mg}$ dose. Copper was repleted initially with copper gluconate $6 \mathrm{mg}$ orally and then changed to a dose of intravenous copper at $2 \mathrm{mg}$ daily for 5 days, followed by $2 \mathrm{mg}$ weekly for 8 weeks and then monthly copper thereafter. She was treated with IVIG at a dose of 2 grams per kilogram divided over 5 days for possible axonal form of Guillain Barré syndrome. Additionally, steroids were given at a rate of $1,000 \mathrm{mg}$ IV daily $\times 5$ days followed by oral prednisone taper for possible inflammatory etiology. She underwent nerve and muscle biopsy for possible vasculitic neuropathy. Her muscle biopsy showed several nonspecific esterase enhanc- ing angular atrophic fibers of acute denervation and minute foci of endomysial inflammatory infiltrate. Her nerve biopsy showed moderate to severe loss of unmyelinated fibers, severe loss of myelinated fibers and severe ongoing Wallerian degeneration, consistent with axonal neuropathy (see Figure 2). She did not notice significant early improvement, but improved slowly over the following two years. Subsequent thiamine level at 1 year was normal, $30.8 \mathrm{nmol} / \mathrm{L}$. Thiamine was checked on a yearly basis. Subsequent copper level was normal at 2 years, $94 \mathrm{nmol} / \mathrm{L}$. Copper was checked on a 6 month to 1 year interval.
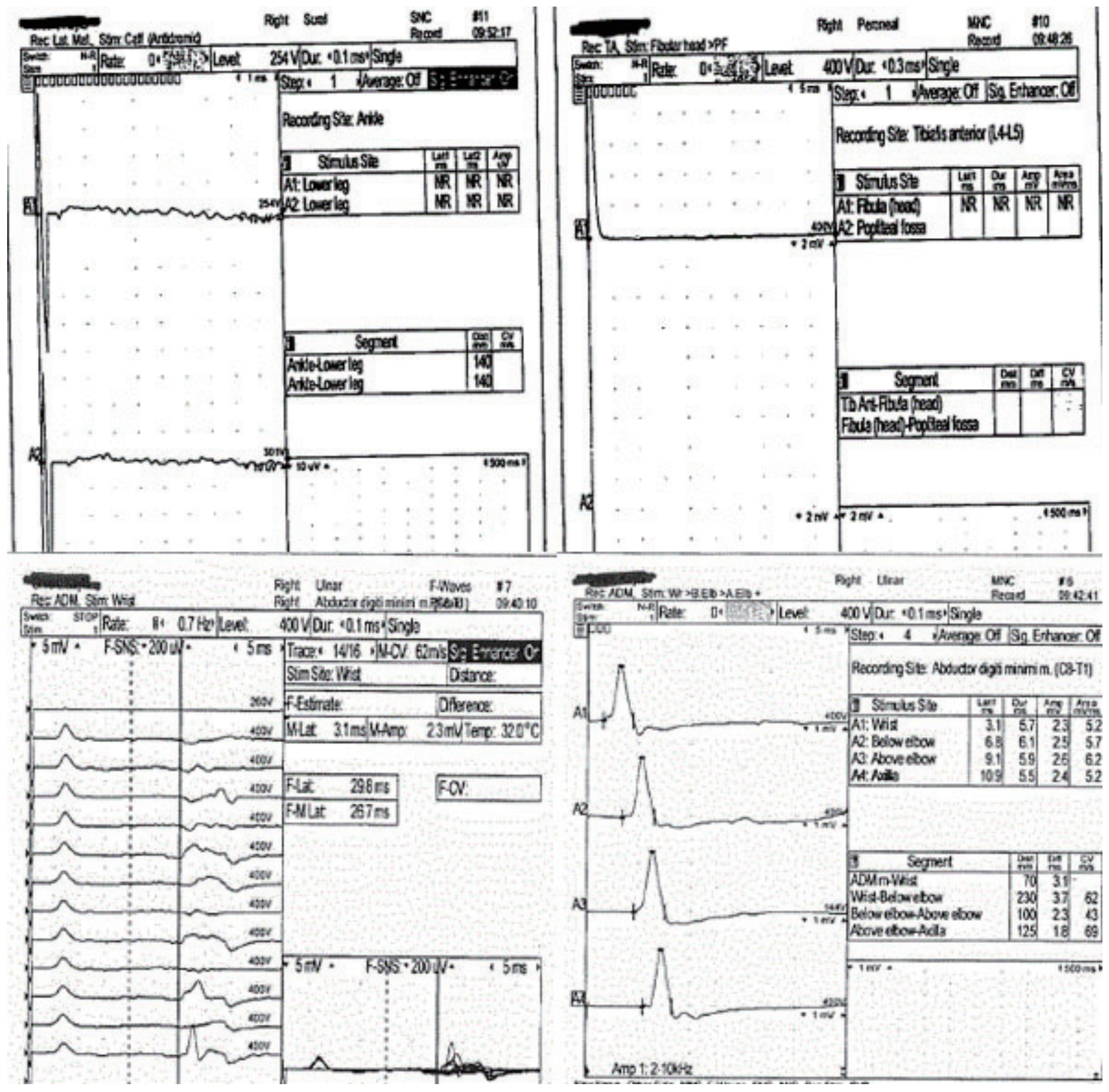

Figure 1. Electrodiagnostic figures show length-dependent, axonal, sensory-motor neuropathy with normal F-waves 


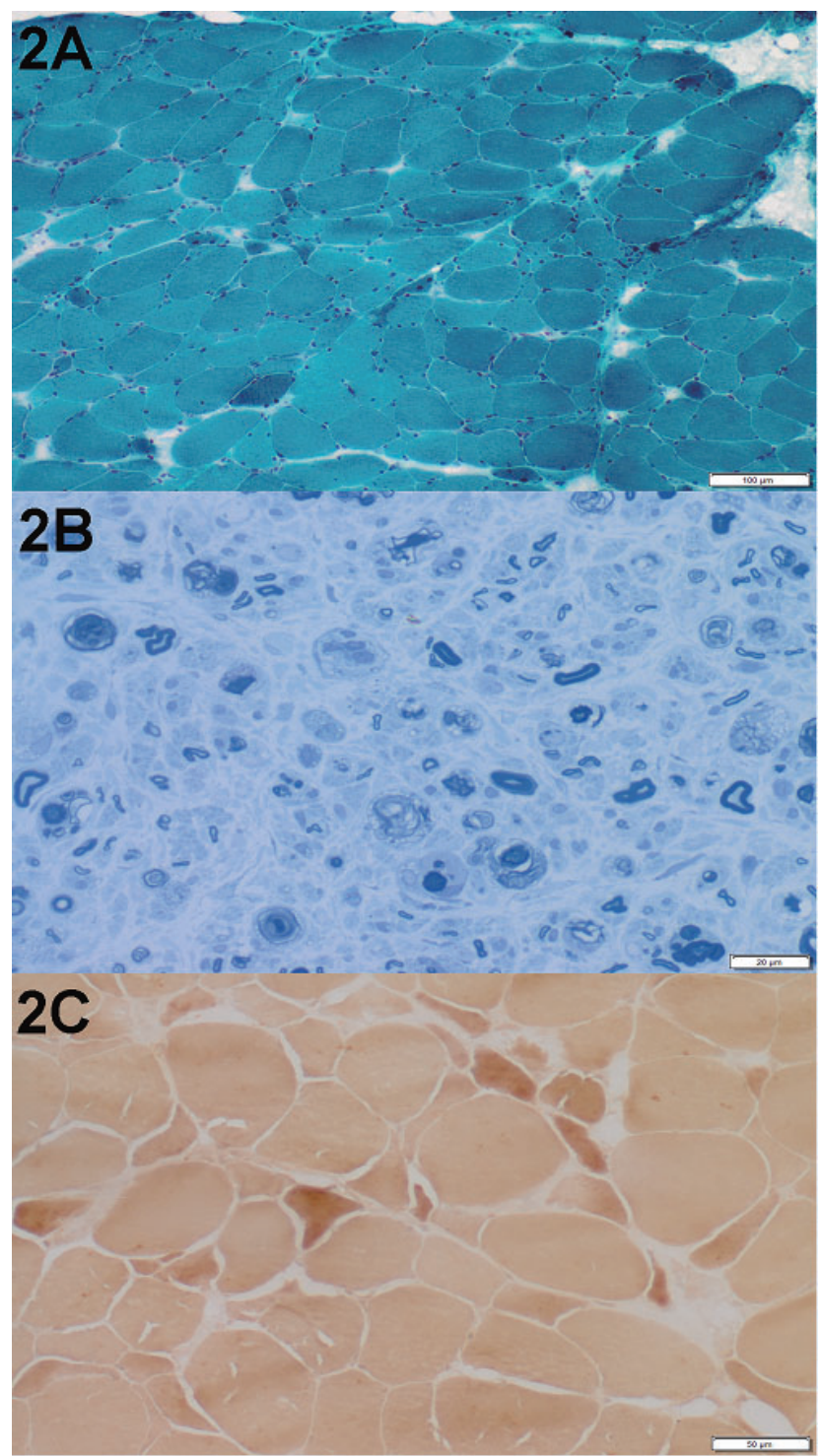

Figure 2. 2A. Modified gomori trichrome stain.

Bar $=100 \mu \mathrm{m}$. Angular fibers and grouped atrophy are noted; 2B. Plastic section of nerve, toluidine blue stain. Bar $=20 \mu \mathrm{m}$. There is active degeneration of multiple nerve fibers undergoing different stages of axonal degeneration. Severe loss of myelinated nerve fibers are noted;

$2 \mathrm{C}$ : Nonspecific esterase stains show angular atrophic fibers are present. Bar=50 $\mu \mathrm{m}$.

\subsection{Case 3}

A 22-year-old woman underwent gastric bypass for obesity. Her surgery was complicated by intractable vomiting. She lost 100 pounds over 4 months. Four months after the surgery, she presented with ascending quadriparesis, numbness and pain over 3 weeks. Neurologic examination showed flaccid, areflexic quadriparesis and length-dependent sensory changes. MRC SS was 30 at nadir during hospitalization. (Bilateral deltoid, elbow flexion, iliopsoas, knee extension were $3 / 5$. Bilateral dorsiflexion was $1 / 5$. Bilateral wrist extension was 2/5.) She required total assistive care from family members at the time of her admission. Electrodiagnostic studies showed severe, presumed axonal sensory-motor neuropathy (all responses absent). Her thiamine level was undetectable $<20 \mathrm{nmol} / \mathrm{L}$ (normal range 78-185 nmol/L). Thiamine was repleted at a dose of 100 milligrams intravenous for 1 day followed by thiamine $125 \mathrm{mg}$ oral dose for 26 days. Patient was lost to follow up to neurology clinic and therefore regular thiamine intervals were not evaluated. One year later, her thiamine level was checked by a different service which showed a low level of thiamine of $56 \mathrm{nmol} / \mathrm{L}$. She did not receive immunomodulatory treatment and was lost to follow up after the initial hospitalization.

\section{Results}

All patients had intractable vomiting and significant rapid weight loss. Additionally, cranial nerves and respiratory muscles were spared. Electrodiagnostic studies showed lengthdependent, sensorimotor axonal neuropathy with absent sural sensory responses in all patient (see Figure 1). CSF studies performed in 2 of 3 patients (patient 1 and 2) did not show albuminocytologic dissociation. MRI spine performed in 2 of 3 patients (patient 1 and 2) showed no evidence of nerve root enhancement and was otherwise non-contributory.

All patients had thiamine deficiency +/- vitamin B6 or copper deficiency (see Table 1). Clinical improvement lagged significantly compared to normalization of nutrient measurements in the blood. Case 1 and case 2 were treated with IVIG as well as nutritional replacement. There was no acute response to IVIG and both patients improved slowly over the subsequent 2 years (see Table 1). Case 3 was lost to follow-up.

\section{Discussion}

Neuropathic disorders are one of the most common complications reported after bariatric surgeries. Neurologists recognize this neuropathy as insidious onset and chronic complication of gastric bypass surgery. However, severe, acute neuropathic disease is under-recognized and associated with significant morbidity. Rapid weight loss, intractable vomiting, gastrointestinal complications and nutritional deficiencies may lead to this form. ${ }^{[1]}$ The presence of significant pain, lack of cranial nerve/respiratory involvement, absence of albuminocytologic dissociation, electrophysiologic findings, and absence of nerve root enhancement on imaging may distinguish these neuropathies from GBS. By the second week, elevated protein in CSF can be seen in $80 \%$ of patients with GBS. ${ }^{[2]}$ Cranial neuropathies occur in up to $62 \%$ of GBS patients. ${ }^{[3]}$ Nerve root enhancement is up to 
$83 \%$ sensitive. ${ }^{[4]}$ Sural sparing pattern carries a $>90 \%$ specificity for GBS, although limited in sensitivity. ${ }^{[5]}$ The electrodiagnostic findings of axonal sensorimotor neuropathy can distinguish these nutritional neuropathies from the demyelinating form of GBS, which is most common in North America. However, electrodiagnostic findings cannot solely rule out axonal variants of GBS or critical illness neuropathy. Therefore, careful analysis of clinical and diagnostic data is paramount. We believe that the improvement in these cases may be due to correction of nutritional deficiencies, rather than immunomodulatory treatment. The differences in our patients compared to the Guillain Barré syndrome patients would include axonal form of neuropathy, lack of albuminocytologic dissociation, lack of nerve root enhancement, prominent pain, protracted recovery and intractable vomiting directly related to gastric bypass procedure. The gradual/protracted recovery over 2 years likely reflects the extent of axonal injury during subacute phase and its subsequent repair.

Nutritional deficiencies after gastric bypass are wellrecognized. ${ }^{[6,7]}$ However, there are no standardized guidelines across centers on vitamin and mineral supplementation. ${ }^{[8]}$ Recently, the importance of thiamine supplementation after gastric bypass has been recognized. The body's stores of thiamine can be depleted within a few weeks. ${ }^{\text {[9] }}$ There are many case reports of Wernicke encephalopathy and thiamine deficiency neuropathy following gastric bypass surgeries in the literature. Most authors suggest thiamine supplementation doses of 100-125 milligrams of thiamine following gastric bypass. However, once thiamine deficiency complications have been established, some authors recommend very high doses of thiamine treatment of up to $500 \mathrm{mg}$ three times daily IV for short periods of time. ${ }^{[10]}$

\section{Conclusions}

It is important to recognize that nutritional neuropathies after bariatric surgeries can present acutely and with severe moribidity. We presented the challenges in distinguishing GBS from this acute nutritional neuropathy. These cases highlight features which may allow distinction between AIDP variant of GBS and acute nutritional neuropathy. However, there is no single factor that allows the exclusion of an axonal variant of GBS. However, the similar features in our 3 cases and the preceding pain prior to the onset of the rapidly progressive weakness may be clues that a nutritional neuropathy may mimic axonal GBS. Careful consideration of clinical presentation, CSF analysis, imaging and electrophysiologic data are important in these patients. In our case series, we were able to identify nutritional deficiencies in all patients. However, prior reports of acute neuropathies occurring after gastric bypass surgery may not have identified any micronutrient deficiencies, which further complicates making this diagnosis. ${ }^{[6,7]}$

When encountering an acutely presenting severe neuropathy in a patient following gastric bypass, the differential diagnosis would include immune-mediated neuropathies (Guillain Barre syndrome), nutritional deficiency neuropathies and some infectious etiologies (west nile virus, tick paralysis). There are no diagnostic guidelines published for this clinical scenario. We would recommend diagnostic studies to include electromyography/nerve conduction studies, lumbar puncture, vitamin serologies, imaging (if clinically indicated), anti-ganglioside antibodies and infectious studies (depending on clinical scenario).

As obesity and gastric bypass surgery rates increase, the incidence of this acute neuropathic complication may increase. Routine screening at 6 month intervals and supplementation of thiamine, vitamin B12, B6, D, E, folate, calcium, magnesium, phosphorus, selenium and copper have been previously recommended. ${ }^{[6]}$ Standardized guidelines for vitamin/micronutrient supplementation across all surgical centers should be implemented.

\section{ACKNOWLEDGeMENTS}

All authors contributed towards the article by making substantial contributions to conception, design, acquisition of data, or analysis and interpretation of data. Thy Nguyen was involved in the conception, design, writing and supervision of the manuscript. Thy Nguyen and Akhila Vijayakumar were involved in the initial draft of the manuscript and data collection. Suur Biliciler and Kazim Sheikh were involved with revisions of the manuscript.

\section{Conflicts of InTERest Disclosure}

The authors declare no competing interests.

\section{REFERENCES}

[1] Thaisetthawatkul P, Collazo-Clavell ML, Sarr MG, et al. A controlled study of peripheral neuropathy after bariatric surgery. Neurology. 2004; 63: 1462-70. PMid: 15505166. http://dx.doi.org/10.
1212/01.WNL.0000142038.43946.06

[2] Van der Meché FG, Van Doorn PA, Meulstee J, et al. GBS consensus group of the Dutch Neuromuscular Research Support Centre. Diagnostic and classification criteria for the Guillain-Barré syn- 
drome. Eur Neurol. 2001; 45: 133-9. PMid: 11306855. http: //dx.doi.org/10.1159/000052111

[3] Bhargava A, Banakar BF, Pujar GS, et al. A study of Guillain-Barré syndrome with reference to cranial neuropathy and its prognostic implication. J Neurosci Rural Pract. 2014; 5: S43-7. PMid: 25540538. http://dx.doi.org/10.4103/0976-3147.145200

[4] Gorson KC, Ropper AH, Muriello MA, et al. Prospective evaluation of MRI lumbosacral nerve root enhancement in acute GuillainBarré syndrome. Neurology. 1996; 47: 813-17. PMid: 8797486. http://dx.doi.org/10.1212/WNL.47.3.813

[5] Derksen A, Ritter C, Athar P, et al. Sural sparing pattern discriminates Guillain-Barré syndrome from its mimics. Muscle Nerve. 2014; 50: 780-4. PMid: 24616124. http://dx.doi.org/10.1002/mus .24226

[6] Koffman BM, Greenfield LJ, Ali II, et al. Neurologic complications after surgery for obesity. Muscle Nerve. 2006; 33: 166-76. PMid:
15973660. http://dx.doi.org/10.1002/mus. 20394

[7] Juhasz-Pocsine K, Rudnicki SA, Archer RL, et al. Neurologic complications of gastric bypass surgery for morbid obesity. Neurology. 2007; 68: 1843-50. PMid: 17515548. http://dx.doi.org/10.12 12/01.wnl.0000262768.40174.33

[8] Mason ME, Jalagani H, Vinik A. Metabolic complications of bariatric surgery: diagnosis and management issues. Gastroenterol Clin N Am. 2005; 34: 25-33. PMid: 15823436. http://dx.doi.org/10.10 $16 / j \cdot g t c .2004 .12 .008$

[9] Aron-Wisnewsky J, Verger EO, Bounnaix C, et al. Nutritional and protein deficiencies in the short term follow both gastric bypass and gastric banding. PLoS One. 2016; 11: e0149588. PMid: 26891123 http://dx.doi.org/10.1371/journal.pone.0149588

[10] Aasheim ET. Wernicke encephalopathy after bariatric surgery: a systematic review. Ann Surg. 2008; 248(5): 714-20. PMid: 18948797. 